\title{
Scarabaeidae (Coleoptera) do Parque Estadual Mata dos Godoy e de área de pastagem, no norte do Paraná, Brasil
}

\author{
Ísis Meri Medri ${ }^{1}$ \\ José Lopes ${ }^{2}$
}

\begin{abstract}
Scarabaeidae (Coleoptera) from Mata dos Godoy State Park and pasture, at north of Paraná, Brazil. The environmental transformations constitute the principal cause of changes in biodiversity decrease. This make the comparative studies between natural and altered environments very important. This work compare the fauna of Scarabaeidae at the following areas: isolated forest fragment and pasture at the secondary stage of succession. Scarabs beetles were collected using four pitfall traps baited with cattle meat in each habitat, fortnightly from November 1998 to April 1999. It was collected a total of 3,113 individuals from 32 species in forest and 956 individuals from 27 species in pasture. The Sörensen index was used to calculate similarity, and Shannon index to calculate diversity. The qualitative similarity found was 0.64 and the quantitative similarity was 0.10 . A relevant difference was found in the diversity of two localities, the value found in pasture was 1.75 and in forest was 2.44 ; these values were significantly different by $T$ test $(p<0.001)$. Our results suggest that the changes in forest area for pasture area caused losses concerning biodiversity of Scarabaeidae and the respective functions of these organisms in the ecosystem.

KEY WORDS. Coleoptera, Scarabaeidae, scavengers, pitfall traps, forest fragment, pasture, decomposition
\end{abstract}

Excrementos e carcaças de animais são recursos alimentares que concentram grande quantidade de energia e por isso são utilizados por vários grupos de artrópodes, entre eles muitos besouros, principalmente os Scarabaeidae.

Os escarabeídeos que utilizam estrume como recurso alimentar, cavam túneis no solo, onde conduzem este material para utilização como substrato para a oviposição e futuro alimento de suas larvas (ESTRADA et al. 1993). Devido a esta atividade, estes besouros exercem importantes funções no ecossistema, tais como a manutenção da fertilidade e a retenção de água no solo (KIRK 1992), o controle sobre ovos e larvas de moscas parasitas presentes no esterco fresco de mamíferos (BERGSTROM et al. 1976 apud ESTRADA et al. 1998); e a atuação secundária na dispersão de sementes e conseqüente participação no processo natural de regeneração de florestas (ESTRADA \& COATES-ESTRADA 1991 apud EsTRADA et al. 1998).

Geralmente, os escarabeídeos coprófagos utilizam também carniça como recurso alimentar e, deste modo, juntamente com os exclusivamente necrófagos, apresentam grande importância na aceleração do processo de decomposição, contribuindo na reciclagem da matéria orgânica (NEALIS 1977 apud KLEIN 1989).

1) Curso de Graduação em Ciências Biológicas, Laboratório de Entomologia, Departamento de Biologia Animal e Vegetal, Universidade Estadual de Londrina. Caixa Postal 6001, Campus Universitário, 86051-990 Londrina, Paraná, Brasil.

2) Departamento de Biologia Animal e Vegetal, Universidade Estadual de Londrina. Caixa Postal 6001, Campus Universitário, 86051-990 Londrina, Paraná, Brasil. E-mail: jea@uel.br 
HALFFTER (1991) constatou que nas florestas tropicais das américas, os besouros generalistas (copro-necrófagos e copro-saprófagos) são predominantes, enquanto que, nas savanas da África, onde ocorre vasto número de mamíferos de grande porte, e conseqüentemente grande suprimento de estrume, os besouros estritamente coprófagos são dominantes, levando-se em conta que nestas áreas a competição por carniça com as aves é muito maior do que nas florestas. A alta freqüência de escarabeídeos generalistas nas florestas tropicais das Américas e Sudeste da Ásia, contradiz a idéia geral de que a fauna das florestas tropicais é primariamente composta de especialistas.

No mesmo trabalho, HALFFTER descreveu que as áreas de pastagens da América tropical, possuem menor diversidade de espécies de Scarabaeidae que as da floresta.

As condições ambientais em uma floresta são amplamente diferentes das de uma área aberta, portanto a destruição dos ambientes naturais para a criação de áreas de pastagem ou de monoculturas provoca alteração nos fatores abióticos, podendo ocasionar com isso, uma mudança na estrutura e na composição das comunidades, que podem sofrer a perda de muitas espécies não adaptadas a estas novas condições ambientais.

A sensibilidade dos coleópteros coprófagos e, em especial, dos Scarabaeinae às mudanças de hábitat, se deve à baixa capacidade de dispersão da maioria das espécies, ao requerimento de grandes extensões de bosque para a manutenção de suas populações e à especialização de sua dieta, baseada principalmente em excremento de mamíferos (HALFFTER \& HALFFTER 1989 apud EsCOBAR 1997).

Segundo GoMÉz-POMPA et al. (1972 apud HALFFTER et al. 1992), a acelerada destruição da floresta tropical faz dos estudos sobre os efeitos das atividades humanas sobre a diversidade e composição da estrutura destas comunidades, uma prioridade.

Estes estudos propiciam um importante registro histórico da ocorrência de determinadas espécies num local e num dado espaço de tempo.

Este trabalho teve como objetivos: realizar o levantamento das espécies de Scarabaeidae, atraídas por isca de carne bovina, na floresta primária do Parque Estadual Mata dos Godoy e numa área de pastagem próxima à mata; comparar quantitativa e qualitativamente a fauna de Scarabaeidae destes locais; e ampliar a Coleção Entomológica do Museu de Zoologia, Universidade Estadual de Londrina.

\section{MATERIAL E MÉTODOS}

O levantamento dos escarabeídeos foi realizado no Parque Estadual Mata dos Godoy localizado no norte do estado do Paraná e em uma área de pastagem situada a aproximadamente $900 \mathrm{~m}$ do limite sul da floresta.

Em ambos os ambientes de estudo, foram utilizadas quatro armadilhas de solo, que consistiram em recipientes plásticos de $23 \mathrm{~cm}$ de comprimento e $9,5 \mathrm{~cm}$ de diâmetro, sendo que na bordo superior de cada recipiente foi acoplado um pequeno frasco com tampa perfurada, servindo como porta isca (MEDRI \& LOPES 2001). Em cada armadilha foram colocados $50 \mathrm{~g}$ de carne bovina moída. Como

Revta bras. Zool. 18 (Supl. 1): 135 - 141, 2001 
líquido fixador, foi utilizado $200 \mathrm{ml}$ de formol a 4\%, em cada armadilha, conforme indicado por LOPES et al. (1994). Em ambos os ambientes de coleta, uma das armadilhas não recebeu isca, servindo como testemunha durante o experimento.

As coletas tiveram periodicidade quinzenal, durante o período de 24 de novembro de 1998 a 27 de abril de 1999. Todo o material coletado foi depositado na coleção de entomologia do Museu de Zoologia da Universidade Estadual de Londrina.

Maiores informações sobre o local de coleta, metodologia, testes estatísticos e índices utilizados, podem ser obtidos em MEDRI \& LOPES (2001).

\section{RESULTADOS E DISCUSSÃO}

No Parque Estadual Mata dos Godoy foram coletados 3.113 espécimens de Scarabaeidae, enquanto que na área de pastagem foram obtidos 956.

A distribuição dos espécimens nas diferentes armadilhas instaladas em ambos os ambientes, é demonstrada na tabela I. Durante todo o experimento, duas armadilhas foram encontradas desenterradas, provavelmente por coatis, impossibilitando a obtenção de coleópteros nestas ocasiões.

Tabela I. Scarabaeidae coletados entre novembro/1998 a abril/1999, em armadilhas controles (P1 e M1) e iscadas com carne (P2, P3, P4, M2, M3 e M4) instaladas na Mata dos Godoy (M) e em área de pastagem $(P)$.

\begin{tabular}{ccrrrrrrr}
\hline Datas & P1 & P2 & P3 & P4 & M1 & M2 & M3 & M4 \\
\hline $24 / 11 / 1998$ & 0 & 14 & 6 & 11 & 9 & 39 & 7 & 3 \\
$11 / 12 / 1998$ & 0 & 53 & 41 & 33 & 37 & 146 & 163 & 57 \\
$22 / 12 / 1998$ & 0 & 75 & 18 & 40 & 11 & 147 & 138 & 194 \\
$05 / 01 / 1999$ & 1 & 43 & 66 & 62 & 3 & 130 & 82 & 56 \\
$19 / 01 / 1999$ & 3 & 12 & 33 & - & 6 & 88 & 118 & 31 \\
$02 / 02 / 1999$ & 0 & 16 & 33 & 9 & 4 & 89 & 101 & 58 \\
$17 / 02 / 1999$ & 0 & 37 & 6 & 32 & 6 & 216 & 96 & 98 \\
$02 / 03 / 1999$ & 0 & 69 & 16 & 44 & 1 & 89 & 127 & 90 \\
$16 / 03 / 1999$ & 0 & 46 & 13 & 20 & 4 & 13 & 115 & 63 \\
$30 / 03 / 1999$ & 0 & 36 & 9 & 28 & 4 & 43 & 52 & 106 \\
$13 / 04 / 1999$ & 0 & 7 & 10 & 10 & 12 & 32 & 44 & 39 \\
$27 / 04 / 1999$ & 0 & - & 1 & 3 & 2 & 33 & 63 & 48 \\
\hline Total & 4 & 408 & 252 & 292 & 99 & 1065 & 1106 & 843 \\
\hline
\end{tabular}

(-) Armadilha desenterrada (interferência de animais).

A análise destes resultados demonstrou, através do teste de Friedman, diferença significativa $(\mathrm{P}<0,05)$ entre o total de escarabeídeos coletados em armadilhas testemunhas e iscadas com carne, sendo que não houve diferença significativa nas comparações entre as armadilhas iscadas.

Pelos resultados, nota-se que a carne bovina utilizada como isca, foi um ótimo atrativo para os Scarabaeidae. A distribuição das espécies coletadas, em ambos os ambientes amostrados, está plotada na tabela II.

Nota-se dentre os resultados obtidos, que 16 gêneros e 32 espécies foram provenientes do Parque Estadual Mata dos Godoy e 13 gêneros e 27 espécies resultaram da área de pastagem. 
Tabela II. Scarabaeidae coletados em armadilhas de solo em ambientes de floresta primária e de pastagem adjacente, entre novembro de 1998 a abril de 1999.

\begin{tabular}{|c|c|c|c|c|c|}
\hline Scarabaeidae & $\begin{array}{l}\text { Área de } \\
\text { pastagem }\end{array}$ & Porcentagem & $\begin{array}{l}\text { Mata dos } \\
\text { Godoy }\end{array}$ & Porcentagem & Total \\
\hline Aphodius sp. & 0 & 0,00 & 6 & 0,19 & 6 \\
\hline Aspidolea sp. & 0 & 0,00 & 1 & 0,03 & 1 \\
\hline Ataenius sp. & 24 & 2,51 & 18 & 0,58 & 42 \\
\hline Ateuchus sp. & 0 & 0,00 & 6 & 0,19 & 6 \\
\hline Canthidium aff. trinodosum (Bohemann, 1858) & 1 & 0,10 & 548 & 17,60 & 549 \\
\hline Canthidium dispar Harold, 1867 & 2 & 0,21 & 46 & 1,48 & 48 \\
\hline Canthidium sp. 1 & 1 & 0,10 & 5 & 0,16 & 6 \\
\hline Canthidium sp. 2 & 1 & 0,10 & 0 & 0,00 & 1 \\
\hline Canthon chalybaeum Blanchard, 1843 & 328 & 34,30 & 5 & 0,16 & 333 \\
\hline Canthon latipes Blanchard, 1843 & 1 & 0,10 & 8 & 0,26 & 9 \\
\hline Canthon sp. 1 & 2 & 0,21 & 59 & 1,89 & 61 \\
\hline Canthon sp. 2 & 34 & 3,56 & 0 & 0,00 & 34 \\
\hline Canthon sp. 3 & 1 & 0,10 & 0 & 0,00 & 1 \\
\hline Cartwrightia cartwrighti Cartwright, 1967 & 3 & 0,31 & 0 & 0,00 & 3 \\
\hline Coprophanaeus jasius (Olivier, 1789) & 108 & 11,29 & 180 & 5,78 & 288 \\
\hline Coprophanaeus saphirinus (Sturm, 1826) & 1 & 0,10 & 8 & 0,26 & 9 \\
\hline Deltochilum brasiliense Laporte, 1840 & 0 & 0,00 & 2 & 0,06 & 2 \\
\hline Deltochilum morbillosum Burmeister, 1848 & 0 & 0,00 & 122 & 3,92 & 122 \\
\hline $\begin{array}{l}\text { Deltochilum (Parahyboma) furcatum (Laporte de } \\
\text { Castelnau, 1840) }\end{array}$ & 0 & 0,00 & 275 & 8,83 & 275 \\
\hline Dichotomius ascanius (Harold, 1869) & 0 & 0,00 & 13 & 0,42 & 13 \\
\hline Dichotomius assifer (Eschscholtz, 1822) & 3 & 0,31 & 403 & 12,94 & 406 \\
\hline Dichotomius bechynei Martinez \&Pereira, 1973 & 0 & 0,00 & 1 & 0,03 & 1 \\
\hline Dichotomius carbonarius (Mannerheim, 1829) & 28 & 2,93 & 30 & 0,96 & 58 \\
\hline Dichotomius fissus (Harold, 1867) & 1 & 0,10 & 91 & 2,92 & 92 \\
\hline Dichotomius mormon (Ljungh, 1799) & 3 & 0,31 & 5 & 0,16 & 8 \\
\hline Dichotomius sp. & 0 & 0,00 & 53 & 1,70 & 53 \\
\hline Eurysternus caribaeus (Herbst, 1789) & 1 & 0,10 & 250 & 8,03 & 251 \\
\hline Eurysternus hirtellus Dalman, 1824 & 45 & 4,71 & 0 & 0,00 & 45 \\
\hline Eurysternus parallelus Dalman, 1824 & 29 & 3,03 & 227 & 7,29 & 256 \\
\hline Leucothyreus sp. & 1 & 0,10 & 0 & 0,00 & 1 \\
\hline Ontherus azteca Harold, 1869 & 0 & 0,00 & 5 & 0,16 & 5 \\
\hline Onthophagus aff. bucculus Mannerheim, 1829 & 0 & 0,00 & 1 & 0,03 & 1 \\
\hline Onthophagus sp. 1 & 1 & 0,10 & 0 & 0,00 & 1 \\
\hline Onthophagus sp. 2 & 0 & 0,00 & 1 & 0,03 & 1 \\
\hline Onthophagus tristis Harold, 1873 & 0 & 0,00 & 11 & 0,35 & 11 \\
\hline Paracanthon pereirai Andretta \& Martinez, 1957 & 1 & 0,10 & 92 & 2,96 & 93 \\
\hline Scybalocanthon sp. & 1 & 0,10 & 639 & 20,52 & 640 \\
\hline Trichillum (Eutrichillum) boucomonti Saylar, 1935 & 328 & 34,30 & 1 & 0,03 & 329 \\
\hline $\begin{array}{l}\text { Trichillum externepunctatum Prudhomme de } \\
\text { Borre, } 1886\end{array}$ & 1 & 0,10 & 0 & 0,00 & 1 \\
\hline Uroxys sp. & 6 & 0,63 & 1 & 0,03 & 7 \\
\hline Total de espécimens & 956 & 100,00 & 3113 & 100,00 & 4069 \\
\hline Total de espécies & 27 & & 32 & & 40 \\
\hline $\mathrm{H}^{\prime}$ & 1,75 & & 2,44 & & \\
\hline Cs & & & & & 0,64 \\
\hline $\mathrm{Cn}$ & & & & & 0,10 \\
\hline
\end{tabular}

( $\left.\mathrm{H}^{\prime}\right)$ Índice de Shannon, (Cs) similaridade qualitativa, $(\mathrm{Cn})$ similaridade quantitativa (Sörensen).

Em ambos os ambientes de coleta a maioria das espécies obtidas foi representada por poucos indivíduos (Fig. 1). Conforme HALFFTER (1991) esta distribuição, em que poucas espécies são muito abundantes e muitas espécies são representadas por um número restrito de indivíduos, é característica de florestas tropicais. Embora os resultados obtidos na atual pesquisa mostre esta tendência, o fato de existir a atração pela isca, mascara a constatação de fenômeno desta natureza. 

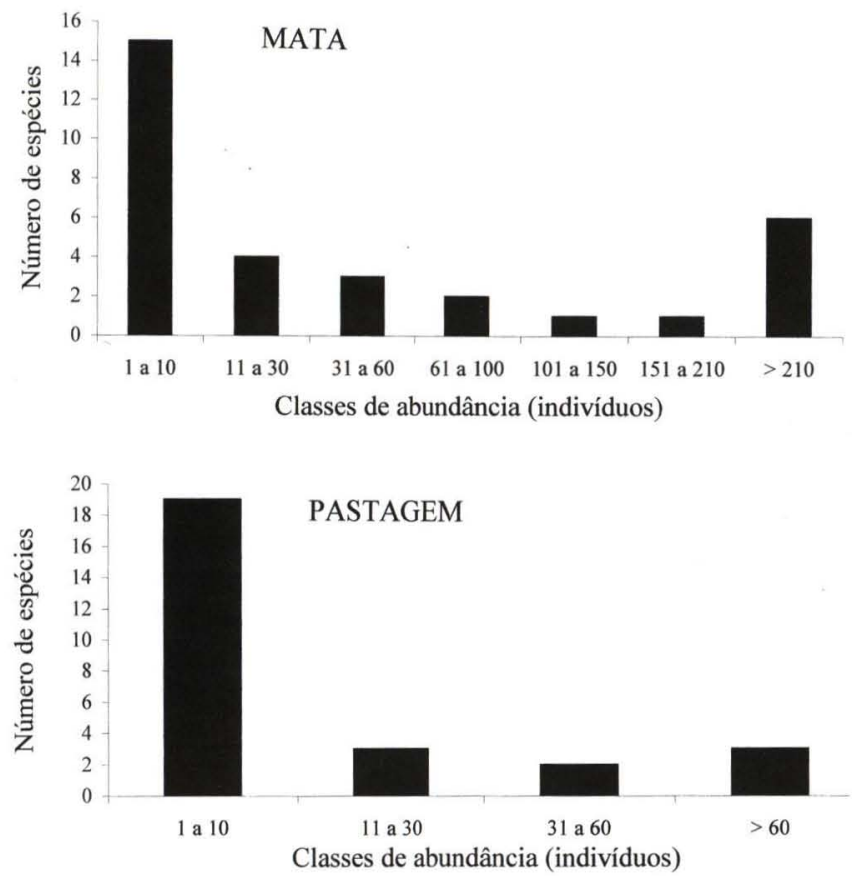

Fig. 1. Número de espécies de Scarabaeidae relacionado ao número de individuos coletados na Mata dos Godoy e em área de pastagem próxima, entre novembro de 1998 a abril de 1999.

No ambiente de floresta primária amostrado o número de escarabeídeos obtido foi maior do que na área de pastagem, provavelmente devido às condições microclimáticas favoráveis e à oferta de recursos alimentares como excrementos e carcaças de animais silvestres e matéria vegetal em decomposição.

Os gêneros Aphodius Illiger, 1798 (Aphodiinae), Aspidolea Bates, 1888 (Rutelinae), Ateuchus Weber, 1801, Deltochilum Eschscholtz, 1822 e Ontherus Erichson, 1847 (Scarabaeinae) ocorreram exclusivamente na floresta, sendo apenas Deltochilum coletado em grande número. Cartwrightia Islas, 1958 (Aphodiinae) e Leucothyreus Macleay, 1819 (Rutelinae), com poucos exemplares, foram exclusivos da área de pastagem (Tab. II). Como os Rutelinae não apresentam hábito coprófago ou necrófago, provavelmente sua captura tenha sido acidental.

As espécies mais abundantes na mata foram: Scybalocanthon sp. (21\%), Canthidium aff. trinodosum (18\%) e Dichotomius assifer (13\%), somando 52\% do total amostrado. Na área de pastagem, Canthon chalybaeum (34\%), Trichillum (Eutrichillum) boucomonti (34\%) e Coprophanaeus jasius (11\%), foram responsáveis por $79 \%$ do total coletado neste local.

Os valores do índice de Shannon resultaram em 2,44 para a mata e 1,75 para o pasto, demonstrando diferença significativa pelo teste $\mathrm{T}(\mathrm{P}<0,001)$. Nota-se, então, diferenças na composição da fauna de besouros de um ambiente natural e de um alterado pela ação antrópica. Esta mudança pode ter sido influenciada pelas 
características do novo ambiente: menor disponibilidade de recursos alimentares como frutos, carcaças e excrementos da fauna silvestre e quase que exclusiva oferta de esterco de ruminantes.

$\mathrm{O}$ índice de Sörensen qualitativo resultou em 0,64 e a similaridade quantitativa foi muito mais baixa, com valor de 0,10 . Deste modo, nota-se que a fauna de Scarabaeidae, dos ambientes analisados, difere mais no número de indivíduos do que na composição, ou seja, a mesma espécie pode ocorrer em ambos os locais mas apresentando uma abundância maior em um dos ambientes.

Alguns dos gêneros obtidos nesta pesquisa também foram coletados por LOPES et al. (1994) no Parque Estadual Mata dos Godoy, durante o período de maio de 1984 a abril de 1985, em armadilhas de solo, com iscas de carne, banana e fezes humanas, além de armadilhas testemunhas (sem isca). No total, coletaram 1.174 espécimens de Scarabaeidae, pertencentes a 42 morfo-espécies. Os gêneros comuns nos dois trabalhos foram: Aphodius (floresta), Ataenius Harold, 1867 (floresta e pastagem), Ateuchus (floresta), Canthidium Erichson, 1847 (floresta e pastagem), Canthon Hoffmannseg, 1817 (floresta e pastagem), Deltochilum (floresta), Dichotomius Hope, 1838 (floresta e pastagem), Eurysternus Dalman, 1824 (floresta e pastagem), Onthophagus Latreille, 1802 (floresta e pastagem) e Trichillum Harold, 1868 (floresta e pastagem).

A fragmentação dos hábitats naturais pode induzir mudanças na abundância e na riqueza de espécies de coleópteros decompositores, podendo causar impactos no suprimento e retenção de nutrientes no solo, na dispersão de sementes e no controle de moscas parasitas de vertebrados e, em conseqüência disso, pode afetar a estabilidade do ecossistema.

\section{CONCLUSÕES}

A carne bovina utilizada no experimento mostrou ser atrativa para os Scarabaeidae, constituindo assim uma eficiente isca para o levantamento desta.

A maioria das espécies de Scarabaeidae coletada nas armadilhas de solo instaladas, foi representada por poucos indivíduos, enquanto espécies como $S c y b a$ locanthon sp., Canthidium aff. trinodosum, Dichotomius assifer e Canthon chalybaeum, foram muito numerosas.

Obteve-se um número três vezes maior de besouros na Mata dos Godoy comparado ao obtido na área de pastagem, o que pode ser devido às condições microclimáticas favoráveis e à disponibilidade de recursos alimentares que a floresta primária oferece, tais como: excrementos e carcaças da fauna silvestre e matéria vegetal em decomposição.

De maneira geral constatou-se, através do índice de Sörensen, alta similaridade qualitativa e baixa similaridade quantitativa, indicando que ocorre maior diferença entre o pasto e a mata no número de escarabeídeos do que na composição destes, ou seja, há diferença nas espécies que são dominantes em cada ambiente.

Em relação à diversidade, os valores resultantes do índice de Shannon demonstraram, pelo teste $T$, diferença significativa na diversidade de espécies de Scarabaeidae das áreas de floresta e de pastagem. 
Portanto, a conversão de floresta para pastagem resulta em perda na diversidade de espécies de Scarabaeidae e conseqüentemente na importante atuação destes organismos no ecossistema.

AGRADECIMENTOS. A Fernando Z. Vaz-de-Mello pela identificação da amostra das espécies de Scarabaeidae coletados; a José Marcelo Torezan pelo auxílio nos cálculos dos índices ecológicos e valiosas sugestões; a Waldir Medri, José Carlos Dalmas e José da Costa Soeiro pelos cálculos estatísticos; a Cláudio Müller pela revisão do artigo; e ao $\mathrm{CNPq}$ pela concessão de Bolsa de Iniciação Científica.

\section{REFERÊNCIAS BIBLIOGRÁFICAS}

ESCOBAR, S.F. 1997. Estudio de la comunidad de coleopteros coprofagos (Scarabaeidae) en un remanente de bosque seco al norte del Tolima, Colombia. Caldasia 19 (3): 419-430.

Estrada, A.; R. Coates-Estrada; A.A. Dadda \& P. Cammarano. 1998. Dung and carrion beetles in tropical rain forest fragments and agricultural habitats at Los Tuxtlas, Mexico. Jour. Trop. Ecol. 14: $577-593$.

Estrada, A.; G. Halffter; R. Contes-Estrada Jr. \& D.A. Meritt. 1993. Dung beetles attracted to mammalian herbivore (Alouatta palliata) and omnivore (Nasua narica) dung in the tropical rain forest of Los Tuxtlas, Mexico. Jour. Trop. Ecol. 9 (1): 45-54.

HALfFTER, G. 1991. Historical and ecological factors determining the geographical distribuition of beetles (Coleoptera: Scarabaeidae: Scarabaeinae). Folia Entomol. Mexicana 82: 195-238.

HALFFTER, G.; M.E. FAvila \& V. HAlfFter. 1992. A comparative study of the structure of the scarab guild in mexican tropical rain forests and derived ecosystems. Folia Entomol. Mexicana 84: 131-156.

KIRK, A.A. 1992. Dung beetles (Coleoptera: Scarbaeidae) active in patchy forest and pasture habitats in Santa Cruz Province, Bolivia, during spring. Folia Entomol. Mexicana 84: 45-54.

KLEIN, B.C. 1989. Effects of forest fragmentation on dung and carrion beetle communities in Central Amazonia. Ecology 70 (6): 1715-1725.

LOPES, J.; I. CONCHON; S.K. YUZAWA \& R.R.C. KUHNLEIN. 1994. Entomofauna do Parque Estadual Mata dos Godoy: II. Scarabaeidae (Coleoptera) coletados em armadilhas de solo. Semina, Londrina, 15 (2): 121-127.

MEdRI, I.M. \& J. Lopes. 2001. Coleopterofauna em floresta e pastagem no norte do Paraná, Brasil, coletada com armadilha de solo. Revta bras. Zool. 18 (Supl. 1): 125-133.

Recebido em 11.V.2000; aceito em 20.VI.2001. 